\title{
RECURSOS EN INTERNET PARA EL ESTUDIO DE LOS ESTADÍSTICOS DE ORDEN
}

\author{
INTERNET RESOURCES TO STUDY ORDER STATISTICS
}

\author{
Silvia Valenzuela \\ Universidad de Granada - España \\ svalenzuela@ugr.es \\ Carmen Batanero \\ Universidad de Granada - España \\ batanero@ugr.es \\ Nuria Begué \\ Universidad de Zaragoza - España \\ nbegue@unizar.es
}

\section{Resumen}

Los estadísticos de orden tienen un papel destacado en el análisis exploratorio de datos y en la inferencia no paramétrica. Su enseñanza comienza en la educación secundaria y continúa en la universidad, niveles en que se han descrito dificultades en el cálculo e interpretación de estos estadísticos. El objetivo de este trabajo es seleccionar y analizar algunos recursos didácticos interactivos que puedan facilitar la comprensión de los estadísticos de orden a los estudiantes de educación secundaria. Se comienza el artículo describiendo nuestros fundamentos, que son el enfoque ontosemiótico del conocimiento e instrucción matemática, el papel de la tecnología en la enseñanza y aprendizaje de la estadística y los resultados de la investigación previa sobre los estadísticos de orden. Una vez descritos el método de selección y análisis de la muestra, se presenta el análisis detallado de tres ejemplos, que comprende su descripción, la identificación de los objetos matemáticos requeridos para el trabajo con los mismos y las posibles dificultades de los estudiantes. Este análisis se complementa con el estudio de las diferentes componentes de la idoneidad didáctica del conjunto de recursos seleccionados y con una lista de otros recursos similares.

Palabras clave: estadísticos de orden, recursos en Internet, análisis semiótico.

\begin{abstract}
Order statistics play a main role in exploratory data analysis and non-parametric inference. Its study is included in secondary education and university, where difficulties in the computation and interpretation of these statistics have been described. The aim of this work is to present and analyse some didactic interactive resources that facilitate the understanding of order statistics to secondary school students. We first describe our framework, which consists in the ontosemiotic approach to mathematical knowledge and instruction, the role of technology in teaching and learning statistics and results of previous research on order statistics. After describing the method of selection and
\end{abstract}


analysis of the sample, we present the detailed analysis of three of these resources, including its description, the identification of the mathematical objects required for working with this tools and possible difficulties of the students. It is complemented by the study of the different components of the resources' didactic suitability and a list of other similar resources.

Keywords: order statistics, Internet resources, semiotic analysis.

\section{INTRODUCCIÓN}

Hoy día la enseñanza de la estadística se extiende a todos los niveles escolares, debido a la necesidad de implicar activamente a los ciudadanos en las decisiones que afectan la salud, política, economía o educación (WATSON; CALLINGHAM, 2003). Se requiere un conocimiento suficiente de lo que se denomina estadística cívica, que permite la participación democrática de los ciudadanos en la sociedad de la información (ENGEL, 2019). De igual modo se insiste en la alfabetización estadística (GAL, 2002), ya que la sociedad está cada vez más dirigida por argumentos basados en datos (WEILAND, 2017).

Un contenido importante en estadística son los estadísticos de orden, que nos indican la posición que un cierto valor de la variable investigada dentro de un conjunto de datos ordenados e informan del porcentaje de datos que tienen un valor de la variable menor o igual que el estadístico (HOAGLIN; MOSTELLER; TUKEY, 1983). Estos estadísticos, que incluyen la mediana, cuantiles, deciles, percentiles y sus órdenes, juegan un gran papel en el análisis exploratorio (TUKEY; 1977), porque están poco afectados por los datos atípicos y son base del gráfico cajas o el gráfico cuantil-cuantil, de gran utilidad al comparar de conjuntos de datos (CHAMBERS, 2018). La mediana es la medida de tendencia central más apropiada para datos que no siguen el modelo de la curva normal, o en variables ordinales. También existen medidas de asociación específicas para variables ordinales basadas en estos estadísticos, como el coeficiente de correlación por rangos de Spearman (ZAR, 2005). Finalmente, son la base de la inferencia estadística no paramétrica, que puede aplicarse en condiciones menos restrictivas que la paramétrica y en pequeñas muestras (VERMA, 2019).

Las directrices curriculares españolas recogen el estudio de la mediana y otros estadísticos de orden en la Educación Secundaria Obligatoria (ESO) y el Bachillerato (MECD, 2015). El cálculo de la mediana y del intervalo mediano aparece por primera vez en el currículo en los cursos primero y segundo de ESO. En tercer curso se contemplan el cálculo, interpretación y propiedades de percentiles y cuartiles, y el gráfico de caja, que se

ReviSeM, Ano 2021, No. 1, p. 1-22 
usa para comparar dos distribuciones en cuarto curso. Los estudiantes también trabajan los estadísticos de orden cuando calculan o representan gráficamente las frecuencias acumuladas. En Bachillerato, los percentiles de la distribución normal se aplican, tanto en la lectura de las tablas de la distribución normal, como en la construcción de intervalos de confianza. Todos estos temas, y la estadística no paramétrica, también se estudian en los cursos universitarios de estadística, tanto de pregrado como de posgrado.

A pesar de esta amplia presencia curricular, algunas investigaciones sugieren la existencia de dificultades frecuentes en estudiantes de ESO (COBO, 2003), Bachillerato (MAYÉN; DÍAZ, 2010; MAYÉN; COBO; BATANERO; BALDERAS, 2007) e incluso en futuros profesores (GEA; BATANERO; FERNÁNDEZ; ARTEAGA, 2016). Esto implica que la sencillez del tema es sólo aparente y podría ser de utilidad contar con recursos didácticos para facilitar su enseñanza. Las nuevas tecnologías facilitan hoy día algunos de estos recursos; en particular, encontramos en Internet numerosos materiales didácticos adecuados para el estudio de diferentes temas matemáticos y que pueden lograr una mayor motivación de los estudiantes (BORBA, 2009). El objetivo de este trabajo es analizar algunos de los recursos didácticos disponibles en Internet y que podrían utilizarse para mejorar la comprensión de los estadísticos de orden por parte de los estudiantes.

\section{MARCO TEÓRICO}

Partimos del enfoque ontosemiótico del conocimiento y la instrucción matemáticos (EOS) (GODINO; BATANERO; FONT, 2007; 2019), que asume que el significado de un objeto matemático es el sistema de prácticas que realiza una persona, o se llevan a cabo en una institución, para resolver las situaciones-problemas de donde surge el objeto matemático; en nuestro caso, los estadísticos de orden. Se diferencia una dimensión personal (subjetiva, mental) y otra institucional (objetiva, epistémica) del significado de un objeto (GODINO, 2002). En nuestro estudio, el significado institucional de los estadísticos de orden será el fijado en los currículos para la Educación Secundaria y Bachillerato, o bien el implementado en un conjunto de recursos didácticos disponibles en Internet, mientras que el significado personal sería el conjunto de prácticas que los estudiantes realizan cuando se enfrentan a los estadísticos de orden. Se asume también la siguiente clasificación de objetos primarios que se usan en la actividad matemática (GODINO et al., 2007):

ReviSeM, Ano 2021, No. 1, p. 1-22 
Campos de problemas: Son las situaciones o aplicaciones que inducen actividades matemáticas de donde surge el objeto. En nuestra investigación, nos interesamos por la clase de problemas asociados a los estadísticos de orden, por ejemplo, encontrar el valor de una variable que deja por debajo un tanto por ciento de la población, comparar dos conjuntos de datos ordinales o resumir gráficamente la distribución de datos utilizando los valores extremos y centrales.

Lenguaje: Los objetos matemáticos son inmateriales y, por tanto, se necesitan representaciones materiales de los mismos (términos, expresiones, símbolos, tablas, gráficos) para poder utilizarlos en la actividad matemática y comunicar esta actividad a otras personas. Por ejemplo, para trabajar con la mediana usamos el término "mediana" o las notaciones $\mathrm{Me}, \mathrm{P}_{50}, \mathrm{D}_{5}$ y $\mathrm{Q}_{2}$.

Algoritmos y procedimientos: Son las acciones y operaciones que se emplean para resolver las situaciones o tareas y que, al llegar a automatizarse, se constituyen en contenido de enseñanza. Se trataría, en nuestro caso, de los diferentes algoritmos de cálculo de los estadísticos de orden o de representación e interpretación de los gráficos asociados. Un ejemplo sería el método para calcular el primer cuartil en un conjunto de datos sin agrupar o en un conjunto de datos agrupados.

Definiciones y propiedades: Ideas matemáticas y sus relaciones (conceptos, proposiciones). Cada estadístico de orden implica un concepto pero, además, hay una serie de propiedades que los relacionan entre sí o con otros conceptos. Así, una propiedad importante de los estadísticos de orden es la robustez que quiere decir que el estadístico apenas varía si en el conjunto de datos se añade un valor atípico.

Argumentos: Finalmente, todas estas acciones y objetos se ligan entre sí mediante argumentos o razonamientos que se usan para comprobar las soluciones de los problemas o explicar a otro la solución, que pueden ser deductivos o inductivos o incluso una visualización puede utilizarse para apoyar un argumento.

Utilizamos también la idea de idoneidad didáctica, que Godino, Contreras y Font (2006) introducen como apoyo en el diseño, desarrollo y evaluación de cualquier proceso de estudio matemático o material didáctico. Godino (2013) hace operativa esta teoría proponiendo un conjunto de indicadores o criterios que permiten valorar los siguientes componentes de la idoneidad didáctica:

ReviSeM, Ano 2021, No. 1, p. 1-22 
Idoneidad epistémica. Grado en el cual el significado institucional implementado en un proceso de estudio de las matemáticas representa al significado institucional pretendido. En nuestro caso, hasta qué punto el significado de los estadísticos de orden en los recursos analizados representa o tiene en cuenta el contenido curricular de estos estadísticos.

Idoneidad cognitiva. Grado de ajuste entre el significado personal del alumno, previo a la instrucción, y el implementado. Implica que los recursos tengan en cuenta los conocimientos previos de un alumno que se enfrenta a su uso, que sean sencillos de utilizar por ellos y que contribuyan a un nuevo aprendizaje.

Idoneidad afectiva. Punto hasta el cual el recurso o actividad analizados son interesantes para el estudiante, promueven en él actitudes positivas, lo implica en el proceso de estudio y le muestran la utilidad y el valor de las matemáticas.

Idoneidad mediacional. Disponibilidad y adecuación de los recursos para su trabajo en el aula y para facilitar el aprendizaje del estudiante en el tiempo disponible.

Idoneidad interaccional. Si el recurso favorece la interacción del alumno con otros alumnos y el profesor y su autonomía en el aprendizaje. Además, estas interacciones deben servir para revelar y resolver las posibles dificultades de aprendizaje.

Idoneidad ecológica. Adaptación del recurso a las directrices curriculares y las condiciones del entorno social y cultural, su utilidad para la innovación docente, así como las conexiones intra e interdisciplinares que se pueden establecer a partir de su uso.

\section{TECNOLOGÍA EN EDUCACIÓN ESTADÍSTICA}

La educación estadística es un área que siempre se ha interesado por la tecnología como apoyo a la enseñanza y aprendizaje, como se analiza, entre otros trabajos, en Biehler, Ben-Zvi, Bakker y Makar (2012) o Pratt, Davies y Connor (2011). En primer lugar, estos medios permiten el trabajo con proyectos estadísticos basados en datos reales, que tienen un interés muy grande para los estudiantes, aumentando su motivación. El uso de la tecnología implica, a la vez, un cambio en la metodología de enseñanza y el contenido de la misma, pues aparecen temas nuevos como el manejo del software (CHANCE; BENZVI; GARFIELD; MEDINA, 2007). Por otro lado, ciertos métodos sencillos de comprender teóricamente (por ejemplo, la correlación) se vuelven dificultosos de aplicar

ReviSeM, Ano 2021, N. 1, p. $1-22$ 
si el conjunto de datos es muy grande, por lo que la tecnología acorta el paso de la comprensión conceptual a la aplicación procedimental (BEN-ZVI, 2000).

Otro segundo apoyo es la simulación y visualización de objetos abstractos, que reduce dicha abstracción al permitir a los estudiantes "manipular" los conceptos y propiedades que se trata de comprender. Esta incorporación en el proceso de enseñanza y aprendizaje resulta de gran utilidad, sobre todo si su preparación matemática no es demasiado alta, y evita tener que reducir la enseñanza a una presentación puramente algebraica (BIEHLER et al., 2012). Este sería el papel de los recursos que analizamos en el trabajo, que están disponibles libremente en Internet para la ayuda de profesores y estudiantes. De hecho, Internet contribuye al autoaprendizaje del estudiante, debido a la variedad y calidad de información disponible sobre cualquier tema y la facilidad de encontrarla, gracias a los motores de búsqueda (BORBA, 2009). Muchos de estos recursos, en especial los applets interactivos, permiten a los estudiantes trabajar sucesivamente con diferentes representaciones de un mismo objeto matemático. Además, de acuerdo con Borba (2009), cambian los tipos de problemas que pueden proponerse y, por ello, el tipo de conocimiento que adquiere el estudiante.

Muchos estudiantes adquieren mejor comprensión de los conceptos estadísticos con la ayuda de imágenes visuales. Es por ello, que se ha producido una gran variedad de applets dinámicos disponibles libres de coste en Internet (PHILLIP, 2003). Estos recursos aumentan la interactividad, es decir, el grado en que los estudiantes tienen control sobre el dispositivo, pueden manipularlo y repetir un proceso o cambiarlo cuando deseen (CAIRNCROSS; MANNION, 2001).

Otro potencial es facilitar el trabajo y comunicación a distancia de los profesores con los estudiantes o de estos entre sí, por medio de videoconferencias o networking. Debido a estas potencialidades, Drijvers (2015) sugiere la necesidad de investigación centrada en la tecnología para analizar el potencial de la misma para el aprendizaje. Nuestro trabajo pretende contribuir a este punto, analizando algunos recursos interactivos que el profesor puede utilizar para favorecer la comprensión de los estadísticos de orden.

\section{ANTECEDENTES DEL TRABAJO}

Aunque no son muchas las investigaciones centradas en la enseñanza de los

ReviSeM, Ano 2021, N. 1, p. 1-22 
estadísticos de orden, encontramos algunas (por ejemplo, COBO; 2003; MAYÉN, 2009) centradas en las medidas de posición central, que incluyen la mediana. A continuación, se resumen los principales trabajos que se centran en la comprensión de estos estadísticos.

Comprensión de campos de problemas. Muchos estudiantes que son capaces de calcular la mediana o un percentil cuando se les pide, no reconocen aquellas situaciones en que deben calcularlos. Así en la investigación de Mayén, Díaz y Batanero (2009), al pedir a estudiantes de Bachillerato elegir la mejor medida de posición central en un conjunto de datos ordinales algunos eligieron la media en vez de la mediana. Este error se repitió en una investigación de Antonio y Mugabe (2013) con estudiantes universitarios y en la de Gea et al. (2016) con futuros profesores de educación secundaria.

Comprensión del lenguaje. Un desafío en la enseñanza de las matemáticas es el uso de un lenguaje apropiado, debido a los diferentes tipos de lenguaje y al hecho de que, en ocasiones, se emplean diferentes símbolos para referenciar un mismo concepto (SCHLEPPEGRELL, 2007). En este sentido, Mayén y Díaz (2010) encontraron estudiantes de ESO y Bachillerato en México que confundían los símbolos usados para la media y mediana o que no eran capaces de identificar la mediana a partir de un gráfico. Igualmente, Bakker, Biehler y Konold (2004) encontraron que el gráfico de la caja, basado en estos estadísticos, es difícil de interpretar por los estudiantes de educación secundaria, debido a las siguiente razones: a) los estudiantes no pueden identificar en el gráfico los casos individuales; b) la mediana no es tan intuitiva como podría esperarse para los estudiantes; c) los cuartiles dividen a la distribución en grupos muy diferentes de lo esperado por los estudiantes; d) el gráfico de la caja no representa frecuencias, sino densidades.

Las definiciones no son siempre claras. Por ello algunos estudiantes de educación secundaria confunden la definición de media y mediana (CARVALHO, 2001; MAYÉN; DÍAZ, 2010). Otros conciben la mediana como centro del conjunto de datos desordenado o incluso como centro geométrico del rango de la variable. Los estudiantes tampoco comprenden el recorrido intercuartílico como medida de dispersión porque asocian las medidas de dispersión a la media y no a los estadísticos de orden (BAKKER; BIEHLER; KONOLD, 2004).

Las propiedades algebraicas propias de las operaciones aritméticas de los estadísticos de orden se generalizan indebidamente; por ejemplo, el tener elemento neutro

ReviSeM, Ano 2021, No. 1, p. 1-22 
o simétrico. Asimismo, al pedir a un grupo de futuros profesores de ESO y Bachillerato interpretar los resúmenes estadísticos de una distribución Gea et al. (2016) informan que algunos interpretaron que todos los elementos del conjunto de datos situados por debajo del primer cuartil tienen el mismo valor de la variable.

Los procedimientos de cálculo son una de las mayores fuentes de dificultad en el trabajo con los estadísticos de orden, debido a la existencia de varios algoritmos de cálculo, dependiendo de si se trata de un conjunto pequeño de datos aislados y si el número de estos es par o impar, de si trabajamos con una distribución de frecuencias y si los datos están o no agrupados en intervalos (SCHUYTEN, 1991). Por ejemplo, la mayor parte de los estudiantes son capaces de calcular la mediana o un percentil de un conjunto pequeño de datos, pero no cuando se les dan organizados en una tabla de frecuencias o a partir de una representación gráfica (SCHUYTEN, 2001).

\section{MÉTODO}

Nuestro estudio puede encuadrarse en el marco de una metodología cualitativa, ya que se analizan objetos (documentos escritos, recursos visuales y applets de Internet) y las variables consideradas (objetos matemáticos implicados en el trabajo con los recursos) son de tipo cualitativo. Se trata de un estudio exploratorio (VASILACHIS, 2019; MAXWELL, 2019), ya que el tema examinado ha sido escasamente estudiado. Existen estudios realizados sobre recursos de internet, pero relacionados con los estadísticos de orden solo hemos encontrado el de Oviedo (2016), centrado en las medidas de tendencia central entre las que se encuentra la mediana. En nuestro trabajo, además de esta medida, se consideran otros estadísticos de orden y se amplía el nivel educativo considerando el Bachillerato, además de la Educación Secundaria Obligatoria.

Se ha realizado un muestreo intencional, que nos ha permitido obtener una muestra reducida de recursos, acorde con el carácter exploratorio y cualitativo de la investigación. Para una primera toma de contacto, se ha optado por analizar un número pequeño de recursos, seleccionados en base a su variedad y su utilidad potencial para la enseñanza del tema. Dichos recursos se seleccionaron de un conjunto más amplio localizado mediante diferentes métodos, también seguidos en otros trabajos que nos anteceden en el análisis de recursos en Internet (GONZÁLEZ, 2017; OVIEDO, 2016; RODRÍGUEZ, 2019; RUIZ,

ReviSeM, Ano 2021, No. 1, p. 1-22 
2013). Las fuentes de donde se han obtenido estos recursos son las siguientes:

- Páginas web de grupos de investigación o sociedades de educación estadística o educación matemática, por ejemplo, International Association for Statistical Education - IASE (https://iase-web.org/), Descartes, Ministerio de Educación (http://recursostic.educacion.es/descartes/web/), NCTM (http://www.nctm.org).

- Localización directa en Internet, utilizando las palabras claves: applet, recurso, material didáctico, vídeo, lecciones, problemas, estadísticos de orden, mediana, percentiles, cuartiles, gráficos de caja, diagrama acumulativo, gráfico cuantilcuantil y sus traducciones al inglés.

Los recursos fueron visualizados y se aplicó a los mismos un análisis de contenido por dos de las autoras, comprobando los resultados para llegar a un consenso y abordando los puntos siguientes: a) Descripción resumida del recurso, incluyendo los contenidos que se pueden abordar y sus posibilidades para adaptarse al trabajo en el aula; b) Análisis semiótico de los objetos matemáticos que intervienen en el trabajo con el recurso; c) Estudio de las posibles dificultades de los estudiantes, comparándolas con las identificadas en los antecedentes; d) Análisis de la idoneidad didáctica del conjunto de recursos analizados.

\section{RESULTADOS}

A continuación se analizan algunos de los recursos didácticos seleccionados, todos ellos útiles para el estudio de los estadísticos de orden en Educación Secundaria Obligatoria y Bachillerato.

\section{Un recurso de exploración}

El primer ejemplo que se ha seleccionado para el análisis es un recurso didáctico que es parte del material didáctico elaborado por Fuensanta Arnaldos mediante Geogebra $\mathrm{y}$ se titula Example of quantiles in a discrete random variable. Podemos encontrarlo en el siguiente enlace: https://www.geogebra.org/m/xz5WWVej. Se trata de un material dinámico con el que se puede explorar el comportamiento de los cuantiles (variando su orden) en variables aleatorias discretas (Figura 1). El recurso permite visualizar gráficamente los cuantiles de una distribución discreta de probabilidad, que se presenta en forma de tabla, 
así como en la función de distribución acumulada. Por tanto, una primera utilidad es diferenciar estas dos funciones de distribución.

En la parte izquierda de la pantalla de inicio encontramos unos ejes cartesianos en los que viene representada la gráfica elegida, en la que se presentan dos iconos móviles que se posicionan según el cuantil que queramos calcular. El primero de ellos, es una línea horizontal discontinua azul que se desplaza verticalmente y representa el orden del cuantil. El segundo, es una flecha morada que apunta al eje horizontal y muestra el valor exacto del percentil con un punto azul. Debajo de este valor aparece una descripción del significado del cuantil calculado.

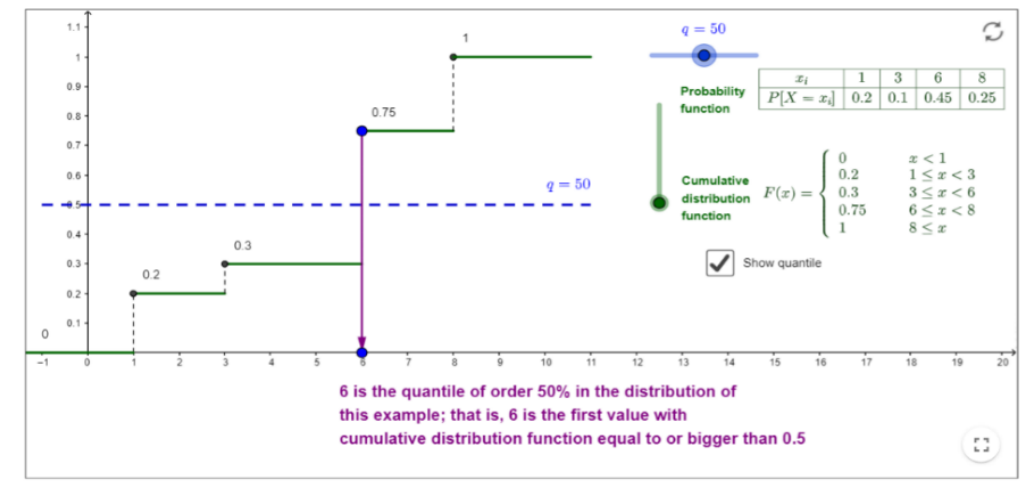

Figura 1. Ejemplo E1: Recurso de exploración de estadísticos de orden Fuente: Materiales de Fuensanta Arnaldos: https://www.geogebra.org/u/fuensanta+arnaldos

En la parte derecha de la pantalla hay otros dos puntos móviles situados cada uno sobre un segmento. Moviendo el punto situado sobre el segmento horizontal (azul) podemos indicar el orden del cuantil que nos interesa representar (por defecto se representa la mediana) y desplazando el punto situado sobre el segmento vertical (verde) indicamos la función que queremos seleccionar. Se utiliza como ejemplo una variable cuya distribución de probabilidad viene representada en una tabla, que se complementa con su correspondiente función de distribución acumulada. Además, se puede elegir representar o no en la gráfica el cuantil seleccionado. Se puede recomenzar cuantas veces se desee el proceso, pulsando las flechas de la esquina superior derecha.

Este recurso nos permite identificar visualmente los distintos cuantiles en variables aleatorias discretas y puede servir como refuerzo para entender los procedimientos analíticos de cálculo y visualizar algunas de sus propiedades. Por ejemplo, se puede discutir con los estudiantes el caso de indeterminación y el hecho de que la función de distribución 
acumulada tome forme de escalera.

\section{Elaboración e interpretación del gráfico de caja}

Otro tipo de materiales disponibles en Internet ayudan en la construcción de gráficos basados en los estadísticos de orden. Un ejemplo, del que reproducimos una de sus pantallas en la Figura 2, es el gráfico de caja de la Red Educativa Digital Descartes, que se puede encontrar en:

https://proyectodescartes.org/iCartesiLibri/materiales_didacticos/EstadisticaProbabilidadInferenci a/Estadistica1D/4_5DiagramasBoxWhisker.html

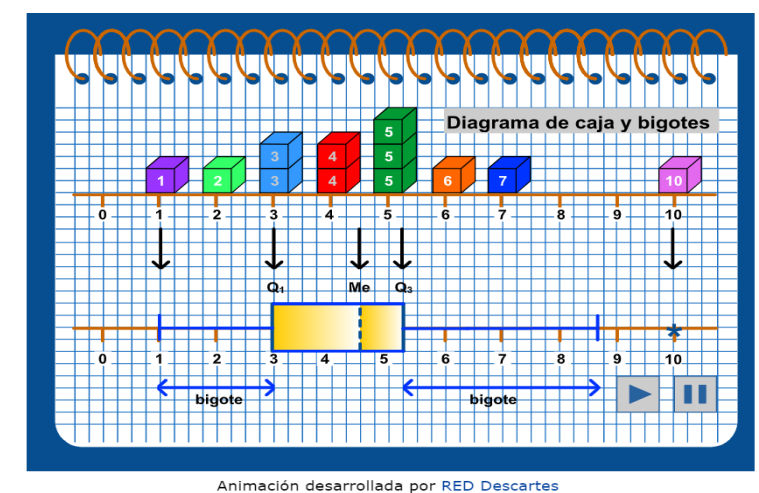

Figura 2. Ejemplo E2: Construcción e Interpretación de gráficos de caja Fuente: Red Descartes, https://proyectodescartes.org/

Este recurso incluye las instrucciones para construir paso a paso dicho gráfico, con una serie de pantallas que guían el proceso. Se comienza proporcionando un pequeño conjunto de datos que hay que ordenar. A continuación, se deben calcular la mediana y los valores máximo y mínimo que se representan sobre una recta. Seguidamente, se calculan el primer y tercer cuartil y aparece un gráfico de caja, pero hay que completarlo situando los extremos de la caja y los bigotes, así como la línea central a la altura de los correspondientes estadísticos que representan. En cada paso se controlan los errores, de modo que no se puede avanzar de un paso a otro hasta que el resultado sea correcto.

Otra serie de pantallas representan nuevos diagramas de cajas acompañados de preguntas cuya respuesta requiere la interpretación de los mismos. Finalmente, se incluye una parte teórica donde se recuerdan y definen los elementos que intervienen en la construcción del gráfico de la caja, incluyendo el significado del valor atípico. Este recurso nos permite trabajar con el gráfico de la caja de forma muy completa, gracias a la 
"animación" que tiene el recurso y que ayuda mucho al estudiante en la visualización del proceso de construcción de la caja y sus bigotes. Se aborda desde su construcción a partir de unos datos (en la que es imprescindible el uso de los procedimientos de cálculo de los estadísticos de orden), hasta la lectura e interpretación de la información proporcionada por el mismo (significado de los estadísticos de orden).

\section{Ayuda en el cálculo}

El tercer ejemplo que presentamos es una ayuda al cálculo de percentiles que, como hemos indicado, es uno de los puntos más difíciles para los estudiantes debido a la existencia de distintos algoritmos. El recurso seleccionado en esta categoría es uno de los materiales proporcionados por Eduardo Timón Moliner, también a partir de Geogebra, que puede obtenerse en el siguiente enlace: https://www.geogebra.org/m/tVutWDF5 y desarrolla el cálculo de los percentiles a partir del diagrama acumulativo de frecuencias absolutas para datos agrupados en intervalos (Figura 3).

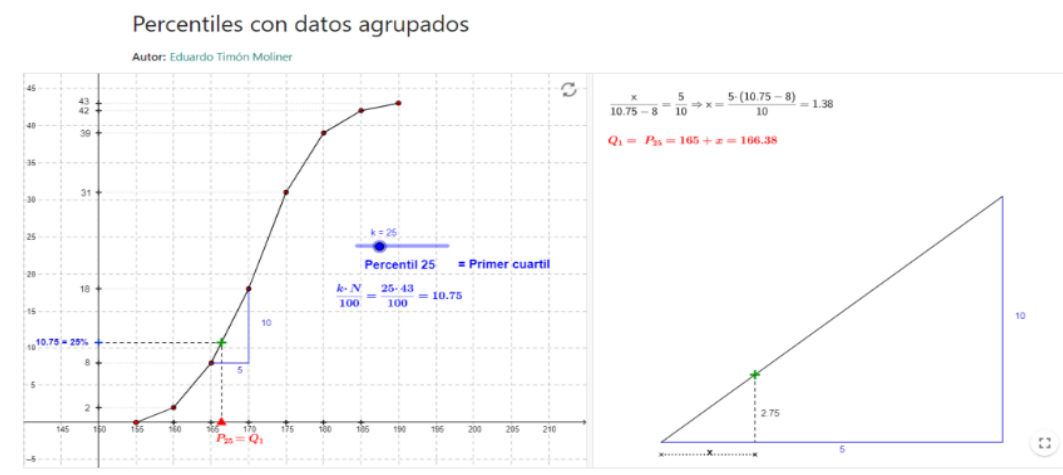

Figura 3. Ejemplo E3: Un recurso de ayuda al cálculo de percentiles

Fuente: Materiales de Eduardo Timón Moliner: https://www.geogebra.org/u/e timon

En la parte izquierda de la pantalla aparece el diagrama acumulativo de frecuencias absolutas, donde se han representado en el eje de abscisas los extremos de los intervalos considerados para la agrupación de los datos. Un triángulo rojo indica el percentil que queremos calcular, junto con su etiqueta correspondiente. En el eje de ordenadas se representan las frecuencias absolutas acumuladas correspondientes a cada intervalo y, en azul, la frecuencia absoluta acumulada correspondiente al percentil indicado en el eje de abscisas. Se puede elegir el orden del percentil que nos interesa mediante un punto móvil 
situado sobre un segmento horizontal. Debajo del mismo, aparece la etiqueta del percentil que corresponde al orden indicado, así como la fórmula y el cálculo de su frecuencia absoluta acumulada.

Cuando los datos se encuentran agrupados en intervalos, generalmente hay que interpolar para encontrar el valor exacto de los percentiles. El procedimiento de interpolación se muestra en la parte derecha de la pantalla de este recurso, en la que se representa un triángulo que amplía el representado en el diagrama de la izquierda. Se debe buscar la distancia x que hay que sumar al extremo inferior del intervalo en que se encuentra el percentil, aplicando proporcionalidad. Este procedimiento se detalla simbólicamente en la parte superior derecha de la pantalla. Una vez calculado $\mathrm{x}$, se determina el valor del percentil (en rojo). En consecuencia, este recurso se puede utilizar para reforzar el aprendizaje del cálculo de percentiles con datos agrupados en intervalos.

\section{Objetos matemáticos implícitos en el uso de los recursos}

Aunque al interaccionar con los recursos el estudiante pudiera actuar de modo mecánico, para poder comprender su funcionamiento en profundidad y utilizarlos posteriormente en su aprendizaje, se deben recordar y utilizar una serie de objetos matemáticos, al menos en forma implícita. En el Cuadro 1 presentamos una lista de los objetos matemáticos requeridos en cada uno de los tres ejemplos de recursos analizados y clasificados según la tipología de objetos que se ha descrito en el marco teórico.

Cuadro 1. Objetos matemáticos implicados en los recursos

\begin{tabular}{|c|c|c|c|c|}
\hline Tipos & Objetos matemáticos & E1 & E2 & E3 \\
\hline \multirow[t]{2}{*}{$\begin{array}{l}\text { Campos de } \\
\text { problemas }\end{array}$} & $\begin{array}{l}\text { Identificar el valor al que corresponde un porcentaje de casos menor o } \\
\text { igual en una distribución. }\end{array}$ & $\mathrm{x}$ & $\mathrm{x}$ & $\mathrm{x}$ \\
\hline & $\begin{array}{l}\text { Resumir la distribución gráficamente mediante casos centrales y } \\
\text { extremos }\end{array}$ & & $\mathrm{X}$ & \\
\hline \multirow[t]{3}{*}{ Lenguaje gráfico } & Función de probabilidad y función de probabilidad acumulada & $\mathrm{x}$ & & \\
\hline & Diagrama acumulativo de frecuencias & & & $\mathrm{x}$ \\
\hline & Gráfico de barras; Gráfico de cajas & & $\mathrm{x}$ & \\
\hline Lenguaje icónico & Puntos para seleccionar las opciones deseadas & $\mathrm{x}$ & & $\mathrm{x}$ \\
\hline \multirow{4}{*}{$\begin{array}{l}\text { Lenguaje } \\
\text { numérico }\end{array}$} & Enteros (valores de la variable) & $\mathrm{x}$ & $\mathrm{x}$ & \\
\hline & $\begin{array}{l}\text { Enteros (extremos de los intervalos, orden del percentil, frecuencias } \\
\text { absolutas acumuladas) }\end{array}$ & & & $\mathrm{X}$ \\
\hline & $\begin{array}{l}\text { Decimales (probabilidades y probabilidades acumuladas, valores de la } \\
\text { escala) }\end{array}$ & $\mathrm{x}$ & & \\
\hline & Decimales (frecuencias relativas y relativas acumuladas) & & & $\mathrm{x}$ \\
\hline
\end{tabular}

ReviSeM, Ano 2021, No. 1, p. 1-22 


\begin{tabular}{|c|c|c|c|c|}
\hline & Porcentajes (orden del percentil) & $\mathrm{x}$ & $\mathrm{x}$ & $\mathrm{x}$ \\
\hline Lenguaje verbal & $\begin{array}{l}\text { Instrucciones de uso. Etiquetas explicativas en la representación } \\
\text { gráfica y términos como función de probabilidad, función de } \\
\text { probabilidad acumulada, cuantil, variable aleatoria discreta. }\end{array}$ & $\mathrm{x}$ & $\mathrm{x}$ & $\mathrm{x}$ \\
\hline \multirow{3}{*}{$\begin{array}{l}\text { Lenguaje } \\
\text { simbólico }\end{array}$} & $x_{i}, X, P\left[X=x_{i}\right], F(x),<,=, \leq$ & $\mathrm{x}$ & & \\
\hline & $x, Q_{1}, Q_{3}, M e, L i, L s,>,<,+,-, \Leftrightarrow, \cdot{ }^{*},()$ & & $\mathrm{x}$ & \\
\hline & $P_{k}, \mathrm{k}, \mathrm{N},=,+,-, \cdot, \%,(), \Rightarrow$ & & & $\mathrm{x}$ \\
\hline \multirow[t]{2}{*}{ Lenguaje tabular } & Tabla de la función de probabilidad & $\mathrm{x}$ & & \\
\hline & Tabla de datos & & $\mathrm{x}$ & \\
\hline \multirow{10}{*}{$\begin{array}{l}\text { Conceptos- } \\
\text { definiciones }\end{array}$} & Variable aleatoria o estadística y valor, mínimo, máximo, rango & $\mathrm{x}$ & $\mathrm{x}$ & $\mathrm{x}$ \\
\hline & Intervalos de valores, extremos & & & $\mathrm{X}$ \\
\hline & Frecuencia acumulada & & $\mathrm{x}$ & $\mathrm{x}$ \\
\hline & Probabilidad & $\mathrm{x}$ & & \\
\hline & \begin{tabular}{|l|} 
Distribución de probabilidad \\
\end{tabular} & $\mathrm{x}$ & & \\
\hline & Distribución de probabilidad acumulada & $\mathrm{x}$ & & \\
\hline & Percentil & $\mathrm{x}$ & $\mathrm{x}$ & $\mathrm{x}$ \\
\hline & \begin{tabular}{|l|} 
Cuartiles, mediana \\
\end{tabular} & $\mathrm{x}$ & $\mathrm{x}$ & $\mathrm{x}$ \\
\hline & \begin{tabular}{|lll}
$\begin{array}{l}\text { Segmento, punto perpendicularidad, paralelismo, longitud, } \\
\text { proporcionalidad, función, rango e imagen }\end{array}$ & & \\
\end{tabular} & $\mathrm{x}$ & $\mathrm{x}$ & $\mathrm{x}$ \\
\hline & Interpolación,Teorema de Thales & & & $\mathrm{x}$ \\
\hline \multirow[t]{3}{*}{ Procedimientos } & Representación gráfica & $\mathrm{x}$ & $\mathrm{x}$ & $\mathrm{x}$ \\
\hline & Lectura e interpretación de tablas y gráficos & $\mathrm{x}$ & $\mathrm{x}$ & $\mathrm{x}$ \\
\hline & Cálculo de percentiles de datos agrupados & & & $\mathrm{x}$ \\
\hline \multirow{8}{*}{$\begin{array}{l}\text { Proposiciones- } \\
\text { propiedades }\end{array}$} & La suma de las probabilidades es 1 & $\mathrm{x}$ & & \\
\hline & Conjunto ordenado de datos & $\mathrm{x}$ & $\mathrm{x}$ & \\
\hline & $\begin{array}{l}\text { El valor de cualquiera de los percentiles debe encontrarse dentro del } \\
\text { rango de valores de la variable estudiada. }\end{array}$ & $\mathrm{x}$ & $\mathrm{x}$ & $\mathrm{x}$ \\
\hline & Relación entre frecuencias y frecuencias acumuladas & $\mathrm{x}$ & & $\mathrm{x}$ \\
\hline & Función creciente del percentil en función de su orden & $\mathrm{x}$ & $\mathrm{x}$ & $\mathrm{x}$ \\
\hline & Valor atípico & & $\mathrm{x}$ & \\
\hline & Propiedades del gráfico seleccionado & $\mathrm{x}$ & $\mathrm{x}$ & $\mathrm{x}$ \\
\hline & Semejanza de triángulos & & & $\mathrm{x}$ \\
\hline \multirow[t]{2}{*}{ Argumentos } & $\begin{array}{l}\text { Observación del gráfico seleccionado y análisis del comportamiento } \\
\text { de los percentiles. }\end{array}$ & $\mathrm{x}$ & $\mathrm{x}$ & $\mathrm{x}$ \\
\hline & Obtener conclusiones con la información proporcionada por el gráfico. & $\mathrm{x}$ & $\mathrm{x}$ & $\mathrm{x}$ \\
\hline
\end{tabular}

Fuente: Elaborado por los autores

Por un lado, cada ejemplo nos permite trabajar con un campo de problemas específico, dos en el caso del segundo ejemplo. Los tres ejemplos utilizan una gran variedad de lenguaje matemático, diferenciándose principalmente en las gráficas utilizadas y también en el uso de diferentes símbolos. Todos ellos utilizan la idea de variable, valores y rangos, así como la de percentil, pero el primer recurso también requiere diferentes ideas sobre la variable aleatoria, su función de probabilidad y distribución y, el tercero, sobre la agrupación de intervalos y sus extremos. Aunque la mayoría de las propiedades coinciden, la idea de valor atípico solo está implícita en el segundo recurso analizado y el tercero se apoya en la semejanza entre triángulos. Este cuadro nos muestra la gran complejidad semiótica del trabajo con estos recursos y, en general, con los estadísticos de orden. El 
profesor debe atender a dicha complejidad y asegurar que el estudiante comprende cada uno de los objetos requeridos antes de sugerir el trabajo con los recursos analizados.

Es también claro que si se logra que el estudiante comprenda los pasos en el trabajo con estos materiales adquirirá sin duda un significado mucho más profundo de los estadísticos de orden, su cálculo e interpretación.

\section{Posibles dificultades de los estudiantes}

El análisis semiótico realizado y resumido en el Cuadro 1 también nos advierte de algunas de las posibles dificultades que el estudiante podría encontrar en su trabajo con los recursos. Comenzando por las ideas de variable, valor y rango, así como su distribución, es importante diferenciar la variable estadística de la variable aleatoria en el ejemplo E1 y, en todos los casos, los conceptos de variable y valor. Además, el estudiante podría confundir frecuencia ordinaria y acumulada, como indican tanto Carvalho (2001) como Cobo (2003), en los ejemplos E2 y E3, y también la probabilidad con la probabilidad acumulada en el ejemplo E1. Igualmente, pueden tener dificultades en cualquiera de los recursos con el trabajo con frecuencias relativas o probabilidades, que se expresan con valores decimales, pues están más acostumbrados a trabajar con números enteros o porcentajes.

Si nos centramos en el ejemplo E1, el alumno podría tener dificultad con el lenguaje al no relacionar los términos cuantil y percentil o el percentil con la probabilidad, ya que la definición de cuantil que da el recurso viene en términos de distribución acumulada y no de porcentaje de casos por debajo del valor del cuantil a la que está acostumbrado el estudiante.

Por otro lado, al estar trabajando con una variable aleatoria discreta, la gráfica de la función de probabilidades acumulada toma forma de escalera y los estudiantes no están acostumbrados a las funciones discontinuas a saltos (ESTEPA, 1994). En el estudio de las funciones, usualmente se trabajan funciones continuas y que se definen mediante una única expresión algebraica, pero en este caso, la expresión algebraica es diferente en distintos tramos del recorrido de la variable. Es también difícil de comprender que, aunque la variable para la cual la función está definida es discreta, la distribución acumulada está definida para una variable continua, es decir, se pasa de una variable discreta a otra continua

ReviSeM, Ano 2021, No. 1, p. $1-22$ 15 
al cambiar de la función de probabilidad a la distribución acumulada.

Además, cuando el rango del cuantil corresponde a una de las mesetas (o conjunto de valores constantes de la gráfica) (en el recurso E1, el cuantil del 30\%), todos los valores de la variable comprendidos en un intervalo cumplen la definición teórica, por lo que el cuantil debiera ser el punto medio del intervalo (en el ejemplo, todos los valores entre 1 y 3). Sin embargo, en el recurso no se señala este caso, sino que se asigna como cuantil el extremo superior del intervalo (valor 3 en el ejemplo). Observamos así un conflicto epistémico en la terminología de Godino et al. (2019), o discordancia entre el significado matemático del cuantil y el significado mostrado en el applet.

Otra dificultad importante puede aparecer si el estudiante no logra el nivel requerido de lectura de gráficos. Así, por ejemplo, Friel, Curcio y Bright (2001) consideran diferentes niveles, que van desde la lectura literal (nivel 1), la capacidad de comparar conjuntos de datos en el gráfico (nivel 2), e interpolar o extrapolar (nivel 3) hasta la lectura crítica (nivel 4). Si el estudiante no logra los últimos niveles puede resultarle compleja la interpretación de los gráficos utilizados en los tres ejemplos y la obtención de conclusiones que se pide en el ejemplo E2.

En cuanto al cálculo de los percentiles presentado en el ejemplo E3, el estudiante puede tener dificultad para diferenciar cuándo debe aplicarse el método de cálculo mostrado (variables con datos agrupados en intervalos) y no otro, así como al realizar la interpolación, aplicando la proporcionalidad requerida en forma indebida (SCHUYTEN, 2001).

\section{Idoneidad didáctica de los ejemplos analizados}

El análisis presentado permite proporcionar algunas indicaciones sobre las diferentes componentes de la idoneidad didáctica de los recursos seleccionados para su posible uso en ESO y Bachillerato:

Idoneidad epistémica: el conjunto de recursos seleccionados presenta alta idoneidad epistémica ya que, como se muestra en el Cuadro 1, se trabajan muchos contenidos específicos sobre los estadísticos de orden (cuantiles, sus rangos, distribución y gráficas). Se usan diferentes modos de expresión: numérica, verbal, tabular, gráfica y simbólica, y se solicitan argumentos. Por tanto, el significado de los estadísticos de orden

ReviSeM, Ano 2021, N. 1, p. $1-22$ 16 
implementado en el conjunto de recursos representa bien al contenido establecido en los documentos curriculares para la ESO y Bachillerato.

Idoneidad cognitiva: se presenta buena idoneidad cognitiva, debido a que los alumnos, al manejar los recursos, ponen en práctica los conocimientos previos necesarios para su uso que han sido trabajados en cursos anteriores. Los recursos tienen un manejo sencillo para el alumno y contribuyen al aprendizaje del tema, cada uno en un punto particular. Así, mientras el segundo refuerza su conocimiento del gráfico de la caja y sus elementos, el primero y el tercero contribuyen a la comprensión de un procedimiento de cálculo concreto.

Idoneidad interaccional: los recursos pueden utilizarse de forma aislada, para trabajar en grupo o bien el profesor con un único ordenador que se proyecte a la clase. Será responsabilidad del profesor organizar adecuadamente la interacción en cada momento, para identificar las dificultades de los estudiantes y ayudarles a superarlas.

Idoneidad afectiva: se promueve el aprendizaje autónomo del tema, pues el estudiante puede utilizar el recurso por sí solo y variar como desee el conjunto de opciones para adaptar su uso a su aprendizaje, lo que influye en su autoestima. Los estudiantes tienen, en general, gran interés por la tecnología, que puede extenderse por ello a la matemática en general.

Idoneidad mediacional: se trata de recursos que facilitan al estudiante la visualización y el estudio de los estadísticos de orden y están disponibles sin costo en Internet. Además, el tiempo de uso se puede adaptar para cada estudiante.

Idoneidad ecológica: los recursos permiten trabajar contenidos incluidos en el currículo de ESO y Bachillerato (MECD, 2015) y son innovadores, pues ofrecen muchas herramientas para abordar el estudio del tema. Permite conectar los estadísticos de orden con las funciones y gráficos y conceptos geométricos. Se relaciona con la sociedad actual, tan ligada a la tecnología.

\section{Otros recursos similares}

Para completar nuestra exposición de resultados, en el Cuadro 2 mostramos una lista de otros recursos similares. Análogamente al ejemplo E1 estudiado, estos nuevos recursos permiten explorar los estadísticos de orden, y sus propiedades, por ejemplo, la 
diferencia entre mediana y moda, el concepto de valor atípico. Estos recursos atienden principalmente a la parte conceptual. Otros, como en el ejemplo E2, introducen diversos gráficos basados en los estadísticos de orden, como la gráfica de frecuencias acumuladas, el gráfico de la caja y el gráfico cuantil- cuantil y proponen actividades basadas en ellos. Contribuyen a reforzar el lenguaje gráfico asociado al tema. Un último grupo de recursos son ayudas al cálculo de estos estadísticos y también refuerzan la comprensión de la parte procedimental de este tópico.

Cuadro 2. Otros recursos útiles para el trabajo con los estadísticos de orden

\begin{tabular}{|c|c|}
\hline Título & Dirección web \\
\hline Box plot & http://www.shodor.org/interactivate/activities/BoxPlot/ \\
\hline Box grapher & http://www.imathas.com/stattools/boxplot.html \\
\hline $\begin{array}{l}\text { Cálculo de la mediana para datos } \\
\text { agrupados }\end{array}$ & https://www.uv.es/ceaces/scrips/tablas/mediana.htm \\
\hline $\begin{array}{l}\text { Cumulative frequency graph and box } \\
\text { plot }\end{array}$ & https://www.geogebra.org/m/Um4hruNu \\
\hline Cumulative distribution function $\mathrm{F}(\mathrm{x})$ & https://www.geogebra.org/m/DYsHpS63 \\
\hline Cumulative distribution and box plot & https://www.geogebra.org/m/Um4hruNu \\
\hline $\begin{array}{l}\text { Desarrollo tablas de frecuencias: } \\
\text { ratio, moda, mediana y percentiles }\end{array}$ & https://www.uv.es/ceaces/scrips/tablf/tafreq4.htm \\
\hline Determination of outliers & https://home.ubalt.edu/ntsbarsh/Business-stat/otherapplets/Outlier.htm \\
\hline Describing and Exploring & https://istats.shinyapps.io/EDA quantitative/ \\
\hline Quantitative Variables & \\
\hline Diagrama de caja & http://dm.udc.es/elearning/Applets/Diagrama_Caja/index.html \\
\hline $\begin{array}{l}\text { Example of quantiles in a discrete } \\
\text { random variable }\end{array}$ & https://www.geogebra.org/m/xz5WWVej \\
\hline Finite order statistics experiment & $\begin{array}{l}\text { http://wiki.stat.ucla.edu/socr/index.php/File:SOCR_Activities_FiniteO } \\
\text { rderStatisticExperiment Chui 051807 Fig1.jpg }\end{array}$ \\
\hline Mean and median & $\begin{array}{l}\text { https://digitalfirst.bfwpub.com/stats applet/stats applet_6_meanmed.h } \\
\text { tml }\end{array}$ \\
\hline Media y Mediana & $\overline{\text { http://geogebra.es/cvg_primaria/05/html/mediana.html }}$ \\
\hline $\begin{array}{l}\text { Mean, median, mode, range } \\
\text { calculator }\end{array}$ & https://www.calculator.net/mean-median-mode-range-calculator.html \\
\hline QQ- plot & https://melbapplets.ms.unimelb.edu.au/?portfolio=qq-plots \\
\hline Q-Q plot Geogebra: & https://www.geogebra.org/m/ETmUIyYU \\
\hline Plot it & http://www.shodor.org/interactivate/activities/PlopIt/ \\
\hline Percentiles y cuartiles & https://www.geogebra.org/m/dxvw7HmZ \\
\hline Percentiles con datos agrupados & https://www.geogebra.org/m/tVutWDF5 \\
\hline Valores atípicos y cajas & https://www.geogebra.org/m/HKeWn6MM \\
\hline
\end{tabular}

\section{CONCLUSIONES}

El objetivo de este trabajo fue mostrar, mediante el análisis de algunos ejemplos, las posibilidades que ofrece Internet hoy día para el aprendizaje de los estadísticos de orden. Como señalan Beltrán-Pellicer, Begué y Roldán (2020), los recursos interactivos de Internet continúan centrando el interés de profesores e investigadores y son utilizados con 
frecuencia en la formación continua del profesorado. Aunque los recursos concretos presentados pudieran ser reemplazados por otros, el método de análisis mostrado y el uso sugerido de los mismos será válido para examinar futuros materiales relacionados con el tema que hemos elegido.

El análisis realizado muestra que la sencillez de los ejemplos estudiados es solo aparente, ya que su empleo necesita muchos objetos matemáticos que el alumno debe dominar o al menos comprender intuitivamente. De otro modo, el trabajo con los recursos no aporta el beneficio esperado y esta conclusión puede extenderse a muchos de los innumerables materiales para la enseñanza de la matemática disponibles en Internet. Es importante entonces que los profesores sean conscientes de esta complejidad y la tengan en cuenta al seleccionar cualquier material para su uso en el aula.

Nuestro trabajo es limitado, pues la extensión solo ha permitido analizar tres de los muchos recursos disponibles, por lo que sería necesario completar el análisis con los de otros similares y también otro tipo de recursos disponibles en Internet, como vídeos educativos o lecciones sobre estadísticos de orden. Sin embargo, pensamos que el trabajo aporta información al profesor, tanto para mostrarle algunos de los recursos disponibles, como al proporcionar la herramienta de análisis semiótico de objetos matemáticos relacionados con el uso de los recursos. Dicho análisis debe ser una preocupación habitual en el profesor, quien debe ser consciente de la posible dificultad de los estudiantes al trabajar con las tareas que se le proponen.

Agradecimientos: Proyecto PID2019-105601GB-I00 / AEI / 10.13039/501100011033 y Grupo FQM126 (Junta de Andalucía).

\section{REFERENCIAS}

ANTONIO, E.; MUGABE, D. O conceito da mediana na perspectiva dos estudantes principiantes. International Journal of Scientific \& Technology Research, Delhi, v., 2, n. 9, p. 202-206, 2013.

BAKKER, A.; BIEHLER, R.; KONOLD, C. Should young students learn about box plots?. En BURRILL, G; CAMDEN. M. (Eds.). Curricular Development in Statistics Education. Sweden: IASE. 2004.

BELTRÁN-PELLICER, P.; BEGUÉ, N.; ROLDÁN, A. Experiencias y recursos TIC en la enseñanza y aprendizaje de la probabilidad. Investigación en Entornos Tecnológicos en 
Educación Matemática, Valencia, v. 1, n.1, p. 15-22. 2020.

BEN-ZVI, D. Toward understanding the role of technological tools in statistical learning. Mathematical Thinking and Learning, Londres, v. 2, n. 1-2, p. 127-155. 2000.

BIEHLER, R.; BEN-ZVI, D.; BAKKER, A.; MAKAR, K. Technology for enhancing statistical reasoning at the school level. En CLEMENTS, M. A.; BISHOP, A.J.; KEITEL, C,; KILPATRICK, J.; LEUNG, F. (Eds.), Third international handbook of mathematics education. New York: Springer. p. 643-689, 2012.

BORBA, M. Potential scenarios for Internet use in the mathematics classroom. ZDM, Berlin, v. 41, n. 4, p. 453-465. 2009.

CAIRNCROSS, S.; MANNION, M. Interactive multimedia and learning: Realizing the benefits. Innovations in Education and Teaching International, Londres, vol. 38, no 2, p. 156-164. 2001.

CARVALHO, C. Interaçao entre pares. Contributos para a promoçao do desenvolvimiento lógico e do desempenho estatístico no $7^{\circ}$ ano de escolaridade. Tesis (Doctorado en Educación). Universidad de Lisboa, Portugal, 2001.

CHAMBERS, J. M. Graphical methods for data analysis, 2. ed. London: Taylor and Francis, 2018.

CHANCE, B.; BEN-ZVI, D., GARFIELD, J.; MEDINA, E. The role of technology in improving student learning of statistics. Technology Innovations in Statistics Education, Los Angeles, Califormia, v.1, n. 1, p. 1-12. 2007. Disponible en: https://escholarship.org/uc/item/8sd2t4rr.

COBO, B. Significado de las medidas de posición central para los estudiantes de secundaria. Tesis (Doctorado en Didáctica de la Matemática). Universidad de Granada, España, 2003.

DRIJVERS, P. Digital technology in mathematics education: Why it works (or doesn't). En $\mathrm{CHO}$, Sung Je. Selected regular lectures from the 12th international congress on mathematical education New York: Springer, p. 135-151. 2015.

ENGEL, J. Cultura estadística y sociedad. En CONTRERAS, J. M.; GEA, M. M.; LÓPEZMARTÍN, M.M.; MOLINA-PORTILlO, E. (Eds.), Actas del Tercer Congreso Internacional Virtual de Educación Estadística. 2019. Disponible en www.ugr.es/local/fqm126/civeest.html.

ESTEPA, A. Concepciones iniciales sobre la asociación estadística y su evolución como consecuencia de una enseñanza basada en el uso de ordenadores. Tesis (Doctorado en Didáctica de la Matemática). Universidad de Granada, España. 1994.

FRIEL, S.; CURCIO, F.; BRIGHT, G. Making sense of graphs: critical factors influencing comprehension and instructional implications. Journal for Research in Mathematics Education 32(2), 124-158. 2001. https://doi.org/10.2307/749671

GAL, I. Adults' statistical literacy: Meaning, components, responsibilities. International Statistical Review, La Haya, v. 70, n. 1, p. 1-25. 2002.

GEA, M., BATANERO, C.; FERNÁNDEZ, J. A.; ARTEAGA, P. Interpretación de

ReviSeM, Ano 2021, No. 1, p. 1-22 
resúmenes estadísticos por futuros profesores de educación secundaria. Journal of Research in Mathematics Education, v. 5, n. 2, p. 135-157. 2016.

GODINO, J. Un enfoque ontológico y semiótico de la cognición matemática. Recherches en Didactiques des Mathematiques, Grenoble, v. 22, n. 2/3, p. 237 - 284. 2002.

GODINO, J. Indicadores de idoneidad didáctica de procesos de enseñanza y aprendizaje de las matemáticas. Cuadernos de Investigación y Formación en Educación Matemática, Costa Rica, Año 8, v. 11, p. 111-132, Dic. 2013.

GODINO, J.; BATANERO, C.; FONT, V. The onto-semiotic approach to research in mathematics education. ZDM, Berlin, v. 39, n. 1, p. 127-135. 2007.

GODINO, J.; BATANERO, C.; FONT, V.. The onto-semiotic approach: implications for the prescriptive character of didactics. For the Learning of Mathematics, 39(1), 38-43. 2019.

GODINO, J.; CONTRERAS, A.; FONT, V. Análisis de procesos de instrucción basado en el enfoque ontológico - semiótico de la cognición matemática, Recherches en Didactique des Mathématiques, Grenoble, France, v. 26, n. 1, p. 39 - 88. 2006.

GONZÁLEZ, M. Análisis de recursos de Internet para la Educación Estadística en el tema de gráficos estadísticos. Tesis (Máster en Didáctica de la Matemática), Universidad de Granada, España. 2017.

HOAGLIN, D.; MOSTELLER, F.; TUKEY, J. Understanding robust and exploratory data analysis. New York: Wiley. 1983.

MAYÉN, S. Comprensión de las medidas de tendencia central en estudiantes mexicanos de educación secundaria y bachillerato. Tesis (Doctorado en Didáctica de la Matemática). Universidad de Granada, España. 2009.

MAYÉN, S.; DÍAZ, C. Is median an easy concept? Semiotic analysis of an open-ended task. In MAKAR, K. (Ed.), Proceedings of the Eighth International Conference on Teaching Statistics. Voorburg, The Netherlands: International Statistical Institute. 2010. Disponible en: http://iase-web.org/documents/papers/icots8/ICOTS8_C265_MAYEN.pdf

MAYÉN, S.; DÍAZ, C.; BATANERO, C. Conflictos semióticos de estudiantes con el concepto de mediana. Statistics Education Research Journal, Dordrecht, v. 8, n. 2, p. 74-93. 2009.

MAYÉN, S.; COBO, B.; BATANERO, C.; BALDERAS, P. Comprensión de las medidas de posición central en estudiantes mexicanos de bachillerato. Unión, 9(1), 187-201. 2007.

MAXWELL, J. Diseño de investigación cualitativa. Madrid: GEDISA. 2019.

MINISTERIO DE EDUCACIÓN, CULTURA Y DEPORTE, MECD. Real Decreto 1105/2014, de 26 de diciembre, por el que se establece el currículo básico de la Educación Secundaria Obligatoria y del Bachillerato. Madrid: Autor. 2015.

OVIEDO, $\mathrm{K}$. Análisis de recursos de Internet para la educación secundaria en el tema de medidas de tendencia central. Tesis (Máster en Didáctica de la Matemática), Universidad de Granada, Granada. 2016.

PHILLIPS, B. Overview of online teaching and internet resources for statistics education.

ReviSeM, Ano 2021, No. 1, p. $1-22$ 
En ENGEL, J. (Ed.). Proceedings, IASE Satellite Conference on Statistics Education and the Internet, Berlin. IASE Disponible en : www.stat.auckland.ac.nz/ iase/publications.php. 2003.

PRATT, D.; DAVIES, N. ; CONNOR, D. The role of technology in teaching and learning statistics. En BATANERO, C.; BURRILL, G.; READING, C. (Eds.), Teaching statistics in school mathematics. Challenges for teaching and teacher education. A Joint ICMI/IASE Study New York: Springer. p. 97-107. 2011.

RODRÍGUEZ, A. Análisis de recursos en internet para la enseñanza de correlación y regresión. Tesis (Doctorado en Didáctica de la Matemática). Universidad de Granada, España. 2019.

RUIZ, K. Análisis de recursos en Internet para la enseñanza de la probabilidad en la educación primaria. Tesis (Master en Didáctica de la Matemática). Granada. Universidad de Granada, España. 2013.

SCHLEPPEGRELL, M. The linguistic challenges of mathematics teaching and learning: A research review. Reading and Writing Quarterly, v. 23, n., p.139-159. 2007.

SCHUYTEN, G. Statistical thinking in Psychology and Education. En D. Vere-Jones (Ed.) Proceedings of the International Conference on Teaching Statistics. Voorburg: The Netherlands: International Statistical Institute. 1991.

SCHUYTEN, G. Research skills: A closely connected triplet of research area, research methodology and statistics. En BATANERO, C. (Ed.), Training researchers in the use of statistics. Granada: IASE. p, 227-230. 2001.

TUKEY, J. Exploratory data analysis. Reading, MA: Addison-Wesley. 1977.

VASILACHIS, I. Estrategias de investigación cualitativa. Madrid: Gedisa. 2019.

VERMA J. Prakash Non-parametric tests for psychological data. En VERMA, J.P. (Ed.), Statistics and research methods in psychology with Excel . Singapore: Springer. 2019, p. 477- 521.

WATSON, J.; CALLINGHAM, R. Statistical literacy: A complex hierarchical construct. Statistics Education Research Journal, Voorburgo, v. 2, n.2, p. 3-46. 2003.

WEILAND, T. Problematizing statistical literacy: An intersection of critical and statistical literacies. Educational Studies in Mathematics, Amsterdam, v. 96, n, 1, p. 33-47. 2017. https://doi.org/10.1007/s10649-017-9764-5

ZAR, J. H. Spearman rank correlation. En COLTON, T; ARMITAGE, P. (eds.), Encyclopedia of Biostatistics, New York: Wiley, p. 7. 2005.

Submetido em 18 de setembro de 2020. Aprovado em 29 de janeiro de 2021.

ReviSeM, Ano 2021, No. 1, p. 1-22 